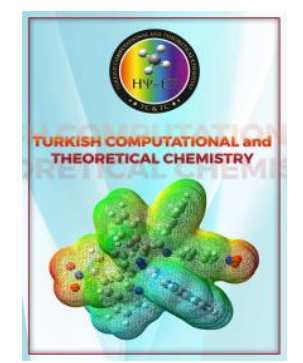

Received: 12.11 .2018
Turkish Computational and Theoretical Chemistry

Turkish Comp Theo Chem (TC\&TC)

Volume(Issue): 3(2) - Year: 2019 - Pages: 59-68

e-ISSN: $2602-3237$

https://doi.org/10.33435/tcandtc.481878

Accepted: 25.02 .2019

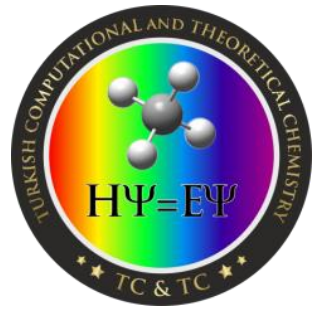

Research Article

\title{
The thermo physical, HOMO, LUMO, Vibrational spectroscopy and QSAR study of morpholinium formate and acetate Ionic Liquid Salts using computational method
}

\author{
Ajoy KUMER ${ }^{1, a}$, Md. Nuruzzaman SARKER ${ }^{\mathrm{b}}$, Sunanda PAUL ${ }^{\mathrm{c}}$ \\ ${ }^{\text {a }}$ Department of Chemistry, European University of Bangladesh, Dhaka-1216, Bangladesh \\ ${ }^{\mathrm{b}}$ Department of Physics, European University of Bangladesh, Dhaka-1216, Bangladesh \\ ${ }^{\mathrm{c}}$ Department of Biochemistry and Molecular Biology, University of Chittagong, Chittagong, \\ Hathazari-4334, Bangladesh
}

\begin{abstract}
Morphine is considered as the uncountable pain killer drug which is taken by both of mouth or injection. In this case, morpholinium ILs is the most applicable molecules due to liquid range so that the thermo-chemical, chemical reactivity and biological interaction of most expected morphonium formate and acetate ILs is considered under theoretical study by HyperChem 8.010 computer programming method. Some thermodynamic parameters such as free energy, entropy, dipole moment, binding energy, nuclear energy, electronics energy, heat of formation and QSAR properties of molecules like charge density, surface area grid, volume, $\log P$, polarizability, refractivity, molecular mass, and reactivity properties of molecule like HOMO, LUMO, HOMO-LUMO, ionization potential and electron affinity were determined using the HyperChem 8.0.10 programme. The morphonium formate is less biological active than morphonium acetate because $\operatorname{LogP}$ is 1.19 and -0.66 respectively. On the other hand, the HOMO LUMO gap in all transition level almost same that indicate similar chemical reactivity. The binding energy of both molecules is -3078.01 and $-3351.25 \mathrm{kcal} / \mathrm{mol}$ respectively. The vibrational spectroscopy data provides them the identification and characterization.
\end{abstract}

Keywords: Morphine, HyperChem, HOMO, LUMO, QSAR, and vibrational spectroscopy.

\section{Introduction}

In the recent time, the computational chemistry is widely used as computers programming to solve real problems in chemical, pharmaceutical, biotechnology and material science with vast popularity of researchers and scientists. It uses the results of theoretical chemistry, incorporated into efficient computer programs, to calculate the structures and properties of molecules and solids materials both of physics and chemistry [1]. It is also entered the numerical problem solving in the design of new drugs and materials. Computational chemistry intense capable of predicting many properties of molecules and chemical reactions, including the following molecular energies and structures, energies and structures of transition states, bond and reaction energies, bond length, bond order, molecular orbitals, vibrational frequencies, thermochemical properties, reaction pathways, spectroscopic quantities and numerous other molecular properties for systems in the gas phase and in solution, including the ground state and excited states [2, 3].

Morphine is recognized as a highly-potent opiate analgesic drug [4] vital active agent in opium and the prototypical opioid [5].

\footnotetext{
${ }^{1}$ Corresponding authors

E-mail: kumarajoy.cu@gmail.com and ajoy@eub.edu.bd
} 
The animated activity of morphine acts directly on the central nervous system (CNS) of human body to relieve pain, and tolerance, physical and psychological dependences develop very rapidly $[6 ; 7]$. In the present time, the morphine is used as the prototype narcotic drug so that it is distributed in the human brain and stay long time with high densities in the posterior amygdala, hypothalamus, thalamus, nucleus caudatus, putamen, and certain cortical areas even was found in the spinal cord and in the spinal nucleus of the trigeminal nerve [8,9], a phenanthrene opioid receptor [10], and gastrointestinal tract acting as the actions of therapeutic value in analgesia and sedation, euphoria, physical dependence, and respiratory depression [11], euphoria/dysphoria, respiratory depression, sedation, pruritus, tolerance, and physical and psychological dependence $[12,13]$. It is unclear, however, what the normal endogenous role of morphine is human body to micro pathogen [14]. Due to the vital role of morphine in pharmaceutical drug and huge impact on human body, the new term was introduced as morphonium based Ionic Liquids as designer molecular in engineering even drug discovery due to tunable thermo-physical and chemical properties [15].

In this study, taking into account all the benefits of designing new drug molecule, to save time and costing, the computational computer programming is used to determine the thermophysical, chemical and biological activity of some morphonium carboxylate ILs salts. The main fact is that some ammonium carboxylate ILs are biological active molecule [16-18]. The chemical reactivity study of ILs gives good evidences for theoretical study in view of HOMO, LUMO and HOMO-LUMO gap study in different level [19,20].

A thermodynamic system is a definite macroscopic region or space in the universe in which one or more thermodynamic processes take place. This system has a specific volume consisting of molecules and atoms with continuous movement and concussion by the interaction with the external surrounding. The internal properties and its interaction with the surrounding determine the system behavior [21].

From above all three, a thermodynamic system of all living organisms is the open system and biological process is the irreversible thermodynamic process where both the change of energy and mass take place. A thermodynamic system when steady state is called an equilibrium system where all the parameters do not vary with respect to time and change by the surrounding which involves transport of the masses. A thermodynamic system can switch from initial state through the intermediate state to final state which is called transformation of state or thermodynamics process $[22 ; 23]$.

\section{Computational Method}

HyperChem 8.0.1is a path of molecular modeling program which permits to build and analyze different molecular structures and determine their physicochemical, thermo-chemical, and biological properties. To performing this work, core i7 computer, and HyperChem 8.0.1 software were done.

The PM3 method is derived from Parametric Method number 3 from computational chemistry and included in the semi-empirical method for the quantum calculation of molecular structure. PM3 was used the Hamiltonian and it is parameterized to reproduce a large number of molecular properties [24].

In order to create the spatial chemical structure of each calculated molecule, the two-dimensional structure of the molecule shall be built step-by-step by drawing. Then hydrogen atoms are automatically added from building option and chemical structure is automatically adjusted from model build option. The first step in getting the main optimized structure, bond length, bond order, and partial charge were taken.

For optimization, MP3 via semi empirical method was ran up and running the option computing using the algorithm Polak-Ribiere was done with maximum gradient set at $0.001 \mathrm{kcal} /$ (mol) for calculation of free energy, entropy, dipole moment, binding energy, nuclear energy, electronics energy, heat of formation, the energy of frontier orbitals, HOMO (Occupied Molecular Orbital Highest) and LUMO (Lowest Unoccupied Molecular Orbital), and QSAR properties of molecules like charge density, surface area grid, volume, LogP, polarizability, refractivity, molecular mass. Using the compute in vibration and spectroscopy, the IR vibration peak was determined. 
Turkish Comp Theo Chem (TC\&TC), 3(2), (2019), 59 - 68

Ajoy KUMER, Md. Nuruzzaman SARKER, Sunanda PAUL

\section{Results and Discussion}

\subsection{Optimized Structure}

A representation of the molecular structural optimization which contains the values of the reactivity indices, are called the reactive molecular diagram. The optimized structures of molecules using the HyperChem 8.0.10 software are provided in fig. 1.The molecular symmetry is a very powerful

\section{IL01}

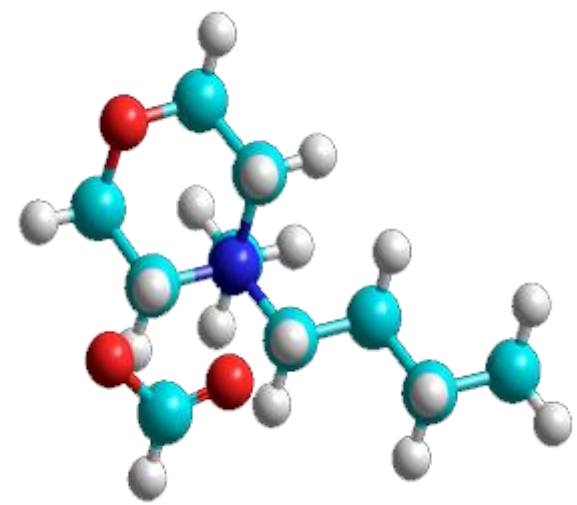

tool established the reactivity computing on the basis of Hyperchem. Using the molecular optimization, it can be found that the both of molecules containing more than one symmetry axis. The molecules belong to the class asymmetry, and non-planar and they have more than one element of symmetry and the plane of the molecule.

Figure 1. Optimized Structure

IL01

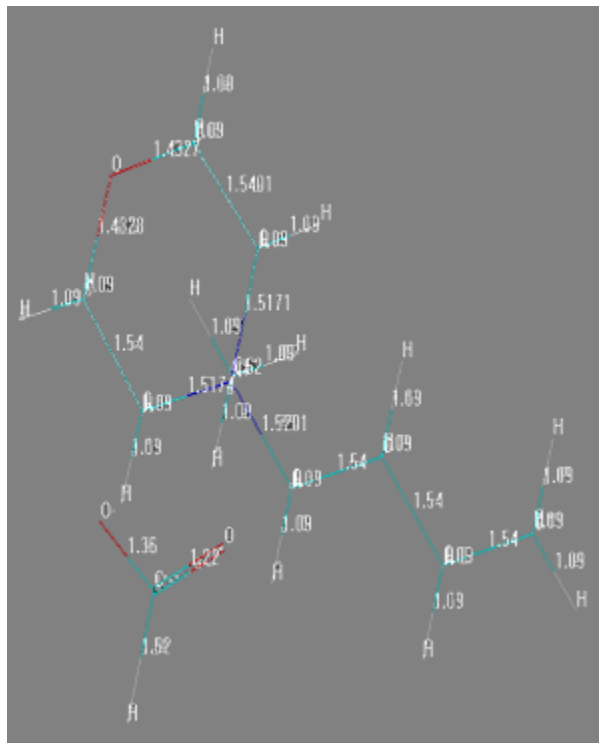

Figure 2. Bond length
$\mathrm{I} 102$



\subsection{Bond length}

Generally, the length of the bond between two atoms is approximately the sum of the covalent radii of the two atoms [25]. For covalent bonds,

IL02



bond energies and bond lengths depend on many factors: electron affinities, sizes of atoms involved in the bond, differences in their electronegativity, and the overall structure of the molecule. There is a 
Ajoy KUMER, Md. Nuruzzaman SARKER, Sunanda PAUL

general trend in that the shorter the bond length, the higher the bond energy. Similar bond length indicates the similarity and molecular symmetry. In the both molecules have same bond length in the oxygen atom of tetrahydralfuran ring almost $1.4327 \mathrm{~A}^{\circ}$.

\subsection{Bond order}

The higher the bond order, are considered as the stronger the pull between the two atoms and the shorter the bond length. The shorter bond length indicates the higher required energy as a result the

\section{IL01}

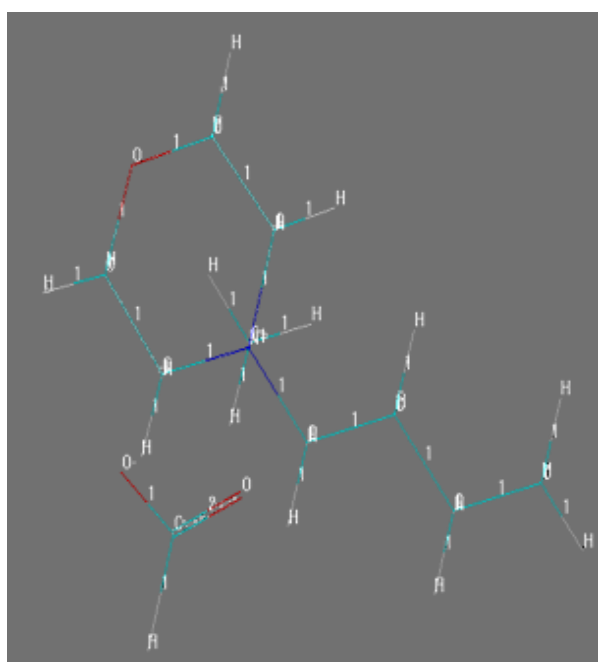

Figure 3. Bond order

\section{IL01}

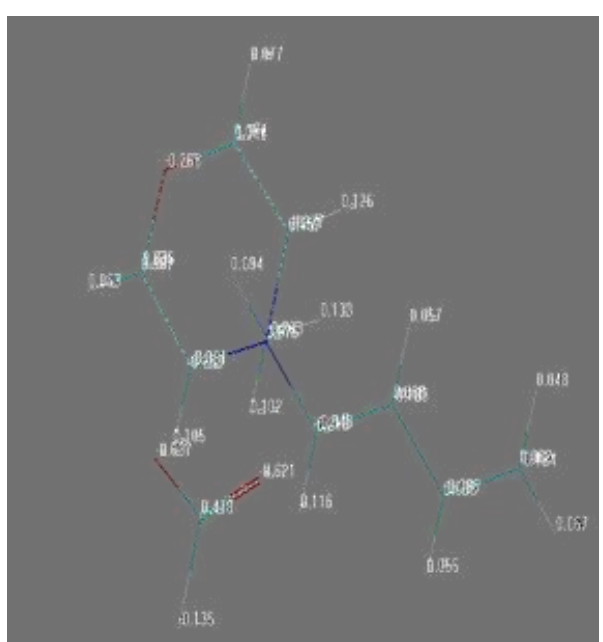

Figure 4. Partial Charge rate of reaction decreases. The oxygen atom of double bonded of carboxylate passes the bond order 2 in both molecules and all other atoms have bond order 1 indicating the strongest pull.

\subsection{Bond order}

Partial charges are created due to the asymmetric distribution of electrons in chemical bonds. All atoms show very little partial charge which indicates the asymmetry distribution of atom in molecules.

\section{IL02}



\section{IL02}






\subsection{Thermophysical Properties}

The binding free energy of the optimized molecules is calculated by performing computing by semiempirical method. The molecule with minimum binding energy will have the maximum binding affinity. The binding free energy of the designed molecules is obtained by eliminating the energy of the main molecule. Having the maximum binding affinity, indicating as the best molecule for drug leads molecules targeting computationally. We can find out the drug binding affinity by using the fitness of the drug, which can bind to the target molecule during the docking process and the second way is using Gibbs free energy calculations. According to this more negative value, we can consider a more effective drug [26]. In our calculation, the value of formate and accetate are 3078.01 and $3351.25 \mathrm{kcal} / \mathrm{mol}$ and mean that accetateanoin has more effective of drug than formate.

The bond dipole moment is the idea of electric dipole moment to measure the polarity of a chemical bond within a molecule. In view of pharmaceutical industries, the dipole moment was a parameter for drug by which a drug is to be used open or close packet. If any drug has dipole moment, it can be able to absorb the ultra violet light and have a possibility of properties. The two optimized ILs show zero dipole moment so that it can be used without any protection from UV or sun light.

Table 1. Thermophysical Properties

\begin{tabular}{lll}
\hline Properties & IL01 & IL02 \\
Total energy, (Kcal/mol) & -58712.1 & -62153.3 \\
Entropy, (Kcal/mol-deg) & 0 & 0 \\
Free energy, (Kcal/mol) & -58712.1 & -62153.3 \\
Heat capacity, (Kcal/mol-deg) & 0 & 0 \\
Dipole moment, $(\mathrm{D})$ & 0 & 0 \\
RMS gradient, $(\mathrm{Kcal} / \mathrm{mol})$ & 3.467 & 2.168 \\
Binding energy, $(\mathrm{Kcal} / \mathrm{mol})$ & -3078.01 & -3351.2563 \\
Heat of formation, $(\mathrm{Kcal} / \mathrm{mol})$ & 16.70 & 18.55 \\
Electronic energy, $(\mathrm{Kcal} / \mathrm{mol})$ & -343767.64 & -391857.86 \\
Nuclear energy, $(\mathrm{Kcal} / \mathrm{mol})$ & 385055.59 & 329704 \\
\hline
\end{tabular}

Table 2. HOMO, LUMO energy data in different energy level

\begin{tabular}{lllllcccc}
\hline \multirow{2}{*}{ IL01 } & Level (-3) & Level (-2) & Level (-1) & Level (0) & Level (1) & Level & Level \\
& & & & & & $(2)$ & $(3)$ \\
& HOMU & -1.84 & -2.32 & -3.15 & -12.21 & -12.57 & -13.89 & -13.96 \\
& LUMO & -13.98 & -12.57 & -12.21 & -3.15 & -2.32 & -1.84 & -1.47 \\
& HOMU- & +12.14 & +10.25 & +9.06 & -9.06 & -10.25 & -12.05 & -12.49 \\
& LUMO gap & & & & & & & -14.41 \\
IL02 & HOMU & -2.20 & -2.67 & -3.58 & -12.64 & -12.97 & -14.36 & -14.41 \\
& LUMO & -14.36 & -12.94 & -12.64 & -3.58 & -2.62 & -2.20 & -1.96 \\
& HOMU- & +12.16 & +10.27 & +9.06 & -9.06 & -10.35 & -12.16 & 12.45
\end{tabular}

\subsection{HOMO-LUMO}

The energy levels of the molecular orbitals order HOMO (Highest Occupied Molecular Orbital) and LUMO (Lowest Unoccupied
Molecular Orbital) for molecules give information on the possible electronic transitional state mention level 1,2,3,0, -1,-2,-3. The HOMO and LUMO also indicate the electrophilic and nucleophilic 
attraction in molecule. The LUMO-HOMO gap is the most important parameter for the chemical reactivity. The shorter LUMO- HOMO gap is considered as the high reactivity, they are highlighted in figure 5 (color: green is positive value and blue is negative value. The electrophilic (Positive charge groups or atoms) attack to the most likely to the atomic site with a high density of orbital HOMO, while nucleophilic (Negative charge groups or atoms) attack LUMO that is
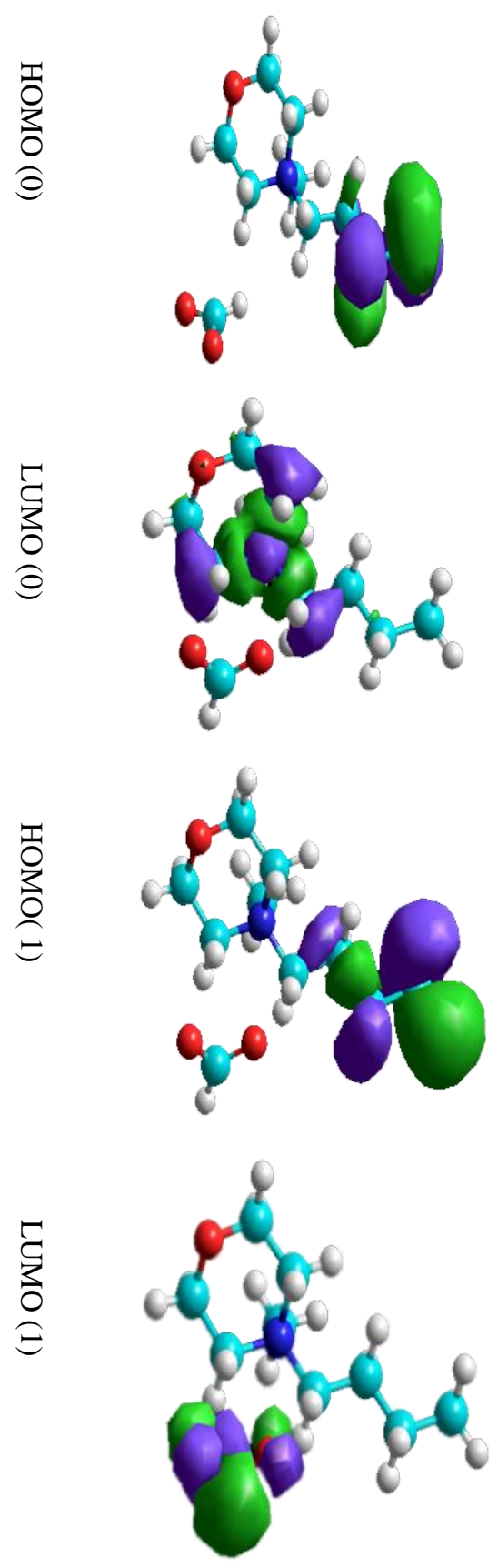

correlated with atomic high-density of orbital LUMO. The ionization potential (I) and electron affinity (A) can be estimated from the HOMO and LUMO energy values like as IP = Negative of the energy of HOMO, and EA = Negative of the energy of LUMO.

\subsection{Orbital Diagram of HOMO LUMO}


Figure 5. HOMO, LUMO molecular orbital diagram in energy level zero and one 


\subsection{The distribution electrostatic potential due to 3D mapped structure}

The stability of the studied molecular structure is given by the higher negative values of total energy. The biological activity of a compound can be estimated on the basis of the energy difference $\Delta \mathrm{E}$ frontier orbitals. This difference, $\Delta \mathrm{E}$ represents the electronic excitation energy which is possible in a molecule. According the mechanism of antimicrobial activity and antimicrobial agents of bioactive molecules, the positive charge end of molecules is responsible to damage the plasma membrane of pathogens [27]. To kill to pathogens, the region of molecules was used the positive charge area of the molecule. In this case the most important factors is explained that the higher surface area having positive charge is considered as the high antimicrobial activity. The electrostatic potential in view of 3D mapped structure indicates positive and negative charge region and charged surface area in a molecule [28] that is considered as the best tools to estimate the biological activity parameters [29].

The surface distribution of molecular electrostatic potential is an indicator of the specific reactive regions of the molecule given in fig-06.
IL01

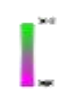

IL02

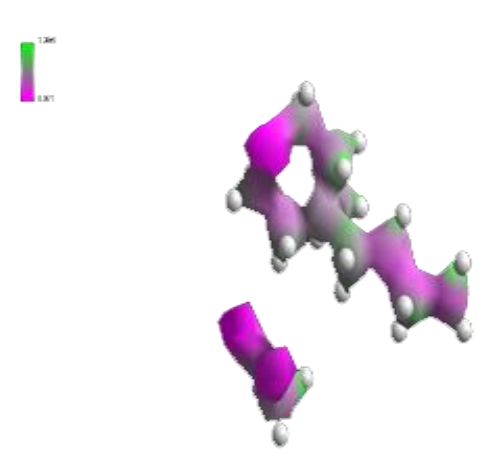

Figure 6. 3D Mapped structure in case of potential energy

\subsection{Vibrational spectrum}

The Vibrational spectra of morphonium based ILs confirmed the presence of carboxylate (COO-) from the symmetric and asymmetric stretching peak at $\sim 1770 \mathrm{~cm}^{-1}$ to $\sim 1550 \mathrm{~cm}^{-1}$ respectively, with the former being overlapped by N-H vibrations. The broad absorption around $\sim 3335-3441 \mathrm{~cm}^{-1}$ can be assigned the presence of $\mathrm{OH}$ groups. The Aromatic ring also be assigned the presence of $\mathrm{C}=\mathrm{C}$ bond stretching of two values at $\sim 1540$ to $\sim 1600 \mathrm{~cm}-1$ and $\sim 1460 \mathrm{~cm}^{-1}$. Then the $\mathrm{C}-\mathrm{O}$ bond is also confirmed the peak of $1260-1000 \mathrm{~cm}^{-1}$ in the carbonate salts. The board peak of $2400 \mathrm{~cm}^{-1}$ to $2700 \mathrm{~cm}^{-1}$ indicates the morphonium ion.

\subsection{Quantitative Structure Activity Relationship (QSAR)}

In case of the biological activity of a molecule, the surface area is considered as the important parameter. Greater charge surface area of a molecule can be able to kill more pathogens. The charged distribution from electrostatic potential completely depends on the surface area. The greater positive charge surface area means the higher biological activity. From the table no-04, it was illustrated that IL01 and IL02 are near in 431.90 and 496.91, where for being a standard drug contains 400 to 500 surface area grids. 
Turkish Comp Theo Chem (TC\&TC), 3(2), (2019), 59 - 68

Ajoy KUMER, Md. Nuruzzaman SARKER, Sunanda PAUL
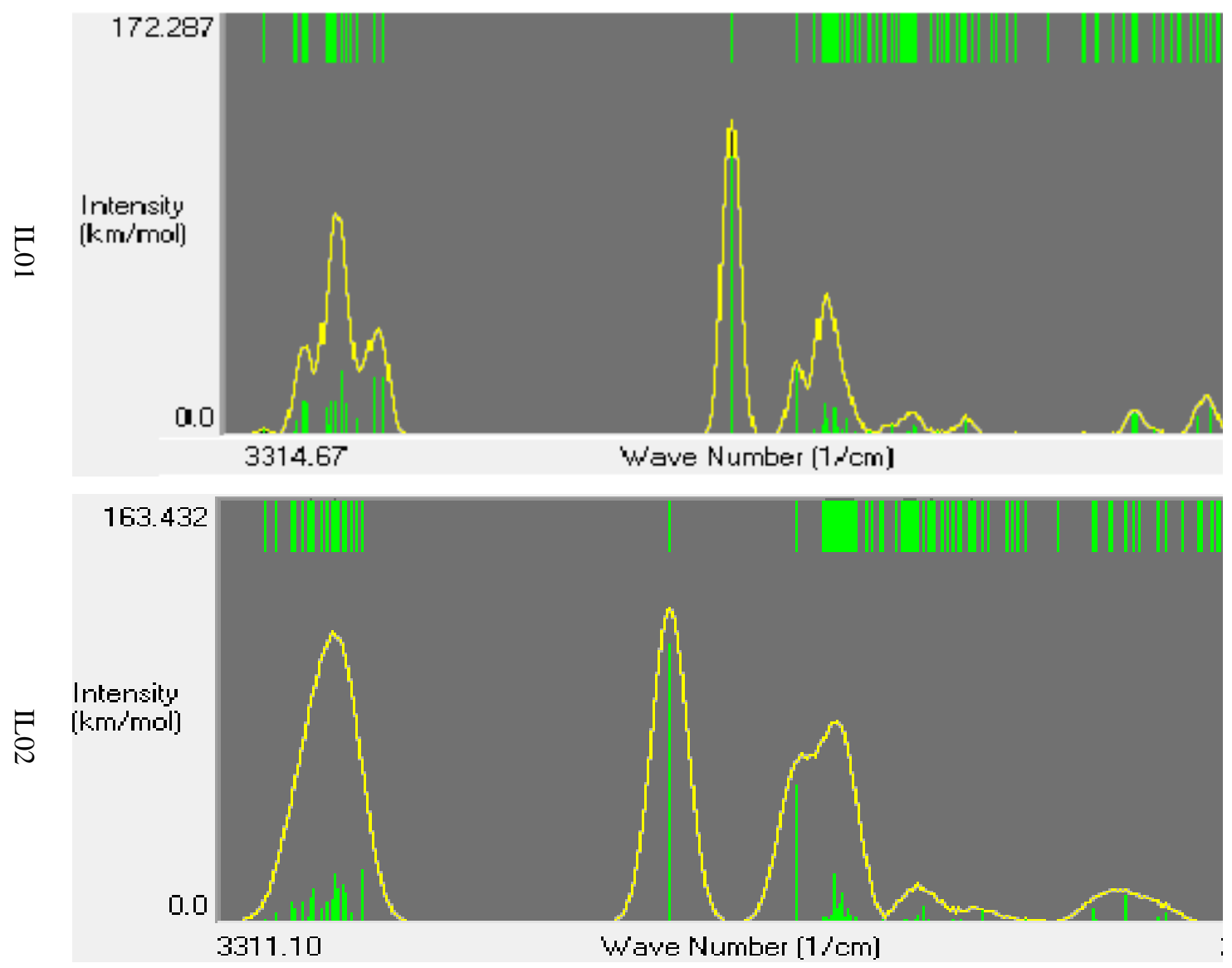

Figure 7. Vibrational spectra of morphonium carboxylate

Table 3. QSAR data of tested molecules

\begin{tabular}{lll}
\hline Partial charge (e) & IL01 & Il02 \\
Surface Area(grid), & 0.0 & 0.0 \\
Volume, $\AA^{3}$ & 431.90 & 496.91 \\
Hydration Energy & 689.53 & 793.51 \\
kcal/mol & -1.06 & 6781.01 \\
Log P & & \\
Refractivity $\AA^{3}$ & 1.19 & -0.66 \\
Polarizibility, $\AA^{3}$ & 54.45 & 58.31 \\
Mass (amu) & 20.80 & 22.64 \\
\hline
\end{tabular}

The hydration energy is defined as the energy absorbed when the substance is dissolved in water. The lower hydration energy is considered as the greater capacity to dissolve in water so that it acts as the hydrophilic nature and predict the best properties of the drug. The molecules of IL01 and IL02, has a great fluctuation of hydration energy where IL01 has $-1.06 \mathrm{kcal} / \mathrm{mol}$ and IL02 can show the $6781.01 \mathrm{kcal} / \mathrm{mol}$.

On the other hand, the biological properties can be explained by $\log$ P term of QSAR optimization. A negative value of $\log \mathrm{P}$ indicates the hydrophilicity and positive $\log \mathrm{P}$ indicates the hydrophobicity that plays an important role in 
biochemical interactions and bioactivity. Hydrophobic drugs tend to be more toxic because, in general, are kept longer, have a wider distribution in the body, are somewhat less selective in their binding to molecules and finally are often extensively metabolized. Therefore ideal distribution coefficient for a drug is usually intermediate (not too hydrophobic nor too hydrophilic). From the data of table no-03, the IL02 has -0.66 values that indicate lower hydrophobicity and IL01 shows hydrophilicity -1.19 .

\section{Conclusion}

The semi-empirical PM3 method of the program HyperChem 8.010 was used to evaluate the thermo-physical properties like total energy, free energy, entropy, dipole moment, formation energy, binding energy, electrostatic energy, and nuclear energy of morphonium formate and acetate ILs salts. The physicochemical parameters and thermodynamic properties were estimated for a specific use to each molecule including 3D structure, bond lengths, the atomic charges.

The most important properties for biological chemistry, chemical reactivity and molecular orbital study like QSAR,HOMO, LUMO, HOMOLUMO gap, ionization potential, electron affinity, and electrostatic potential in case of the charge distribution in molecule were optimized which indicates that it is good drug molecule than morphine.

\section{Acknowledgments}

I am thankful to Prof. Dr. Mukbul Ahmed Khan, V.C of European University of Bangladesh and also thankful to Mr Engr. Zemam Ahmad, Head of IT department and Mr. Moinul Islam, Assistant IT Officer, European University of Bangladesh, for all kind of technical supports.

\section{References}

[1] D.G. Ramachandran K.I., Krishnan Namboori. P.K. -Verlag GmbH ISBN -3540-, Computational Chemistry and Molecular Modeling Principles and applications. Springer 77302-77303.

[2] C. T., A Handbook of Computational Chemistry, in, Wiley, New York, 1985.

[3] W.M. Reichert, Holbrey, J.D., Swatloski, R.P., Gutowski, K.E., Visser, A.E.,
Nieuwenhuyzen, M., Seddon, K.R. and Rogers, R.D., Solid-State Analysis of LowMelting 1,3-Dialkylimidazolium Hexafluorophosphate Salts (Ionic Liquids) by Combined X-ray Crystallographic and Computational Analyses. Cryst. Growth Des 7 (2007) 1106-1114.

[4] L. Martini, J.L. Whistler, The role of mu opioid receptor desensitization and endocytosis in morphine tolerance and dependence. Current opinion in neurobiology 17 (2007) 556-564.

[5] W.D. Bowen, S. Gentleman, M. Herkenham, C.B. Pert, Interconverting $\mathrm{mu}$ and delta forms of the opiate receptor in rat striatal patches. Proceedings of the National Academy of Sciences 78 (1981) 4818-4822.

[6] T.J. Coderre, A.L. Vaccarino, R. Melzack, Central nervous system plasticity in the tonic pain response to subcutaneous formalin injection. Brain research 535 (1990) 155158.

[7] T.J. Coderre, J. Katz, A.L. Vaccarino, R. Melzack, Contribution of central neuroplasticity to pathological pain: review of clinical and experimental evidence. Pain 52 (1993) 259-285.

[8] H.J. Groenewegen, F.T. Russchen, Organization of the efferent projections of the nucleus accumbens to pallidal, hypothalamic, and mesencephalic structures: a tracing and immunohistochemical study in the cat. Journal of Comparative Neurology 223 (1984) 347-367.

[9] D. Van Der Kooy, R.F. Mucha, M. O'Shaughnessy, P. Bucenieks, Reinforcing effects of brain microinjections of morphine revealed by conditioned place preference. Brain research 243 (1982) 107-117.

[10] A. Mansour, H. Khachaturian, M.E. Lewis, H. Akil, S.J. Watson, Anatomy of CNS opioid receptors. Trends in neurosciences 11 (1988) 308-314.

[11] K. Tsou, S. Brown, M. Sanudo-Pena, K. Mackie, J. Walker, Immunohistochemical distribution of cannabinoid CB1 receptors in the rat central nervous system. Neuroscience 83 (1998) 393-411. 
[12] T.L. Yaksh, T.A. Rudy, Analgesia mediated by a direct spinal action of narcotics. Science 192 (1976) 1357-1358.

[13] L.A. Dykstra, D. McMillan, L. Harris, Antagonism of morphine by long acting narcotic antagonists. Psychopharmacologia 39 (1974) 151-162.

[14] I. Izquierdo, Effect of naloxone and morphine on various forms of memory in the rat: Possible role of endogenous opiate mechanisms in memory consolidation. Psychopharmacology 66 (1979) 199-203.

[15] I. Newington, J.M. Perez-Arlandis, T. Welton, Ionic liquids as designer solvents for nucleophilic aromatic substitutions. Organic Letters 9 (2007) 5247-5250.

[16] M.I. Hossain, Kumer, Ajoy, Begum, Sayeda Halima, Synthesis and Characterization of Ammonium Benzoate and Its Derivative Based Ionic Liquids and Their Antimicrobial Studies. Asian journal of physical and chemical science 5 (2018) 1-9.

[17] M.I. Hossain, A. Kumer, Synthesis and characterization of ammonium benzilate bioactive ionic liquids and their antimicrobial activity. Asian Journal of Physical and Chemical Sciences 4 (2017) 1-13.

[18] M.I. Hossain, Kumer, Ajoy, Synthesis and characterization of ammonium ionic liquids and their antimicrobial and computational overview. Asian Journal of Chemical Sciences 3 (2017) 1-10.

[19] A. Kumer, B. Ahmed, M.A. Sharif, A. AlMamun, A Theoretical Study of Aniline and Nitrobenzene by Computational Overview.

[20] A. Kumer, Ahmed, Boshir, Sharif, Md Arfat, Al-Mamun, Abdullah, A Theoretical Study of Aniline and Nitrobenzene by Computational Overview. Asian journal of physical and chemical science, 4 (2018) 1-12.
[21]A.H. Shapiro, The Dynamics and Thermodynamics of Compressible Fluid Flow: In Two Volumes, Wiley, 1953.

[22]E.A. Guggenheim, Thermodynamics-an advanced treatment for chemists and physicists. Amsterdam, North-Holland, 1985, 414 p. (1985).

[23]L. Von Bertalanffy, The theory of open systems in physics and biology. Science 111 (1950) 23-29.

[24] A. Howard, J. McIver, J. Collins, Hyperchem computational chemistry. Hypercube Inc., Waterloo (1994).

[25]R. Mulliken, Electronic population analysis on LCAO-MO molecular wave functions. II. Overlap populations, bond orders, and covalent bond energies. The Journal of Chemical Physics 23 (1955) 1841-1846.

[26] A.R. Ortiz, M.T. Pisabarro, F. Gago, R.C. Wade, Prediction of drug binding affinities by comparative binding energy analysis. Journal of medicinal chemistry 38 (1995) 2681-2691.

[27] L. Timofeeva, N. Kleshcheva, Antimicrobial polymers: mechanism of action, factors of activity, and applications. Applied microbiology and biotechnology 89 (2011) 475-492.

[28] K.D. Collins, Charge density-dependent strength of hydration and biological structure. Biophysical journal 72 (1997) 6576.

[29] M. Böhm, J. Stürzebecher, G. Klebe, Threedimensional quantitative structure- activity relationship analyses using comparative molecular field analysis and comparative molecular similarity indices analysis to elucidate selectivity differences of inhibitors binding to trypsin, thrombin, and factor Xa. Journal of medicinal chemistry 42 (1999) 458-477. 